\title{
Ensayo \\ Playas y espadas de Caravaggio
}

Fecha de recepción : 26 de octubre de 2018 • Fecha de aceptación: 19 de noviembre de 2018 • Fecha de publicación: 7 de enero de 2019

PhD. Armando Valdés Zamora ${ }^{1}$

Universidad de París XII (UPEC) / Escuela Superior de Gestión (ESG) de París, Francia arvelitera@yahoo.fr

$1 \quad$ Armando Valdés Zamora (La Habana, 1964) es doctor por la Universidad de la Sorbona. Autor del poemario Libertad del silencio y de la novela Las vacaciones de Hegel, finalista del premio a la mejor primera novela publicada en Francia en 2003. Ha escrito numerosos artículos y ensayos sobre la literatura cubana. Trabaja como Profesor Adjunto en la Universidad de París XII (UPEC) y de la Escuela Superior de Gestión (ESG) de París. 


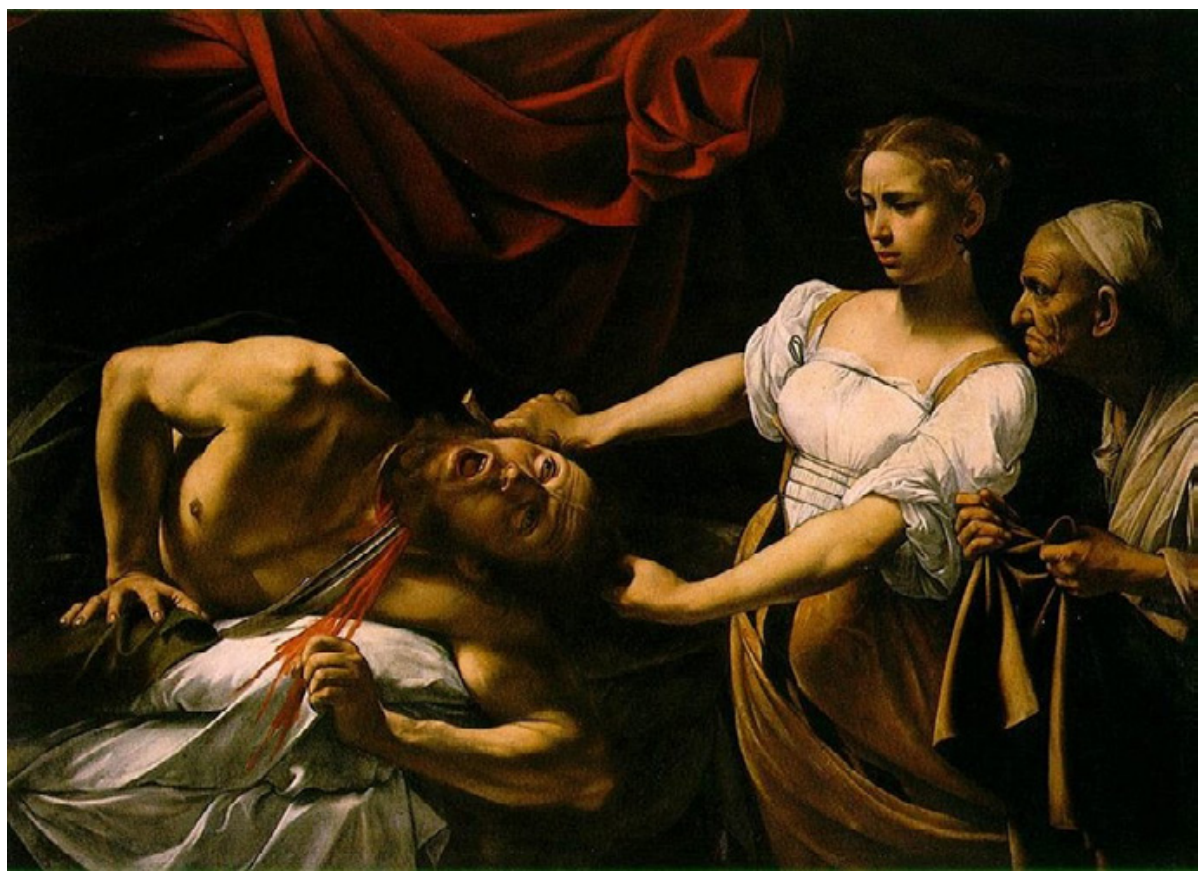

Figura 1. Judith y Holofernes. Caravaggio, M. (1598-1599).

Tomada de https://www.wikiart.org/es/caravaggio/judit-y-holofernes-1599

\section{Playas}

Corriendo, tropezando, cayendo una y otra vez sobre la arena, levantándose apenas mientras grita sin fuerzas por la fiebre de una insolación y las heridas de su más reciente trifulca en Nápoles. Se sabe que murió en una playa de Toscana. La playa de Feniglia en Porto Ercole. Heridas que desfiguran su rostro, que lo hacen irreconocible. Obsesionado con los 3 lienzos de obras maestras (San Giovannino Battista, el San Giovanni disteso y la Magdalena en éxtasis) abandonadas antes de bajar por la fuerza a tierra; premeditados regalos al Cardenal Scipione Borghese, sobrino del Papa Paul V que - él lo ignora- ya lo ha perdonado por su crimen después de un partido de tenis y la deuda de 10 escudos.

Desfallecido al final por tratar en vano de alcanzar la faluca que despliega su vela con sus tres cuadros a bordo hasta perderse de vista en el encandilado horizonte, yace sobre la arena Michelangelo Merisi. Es tomado por un vagabundo más quien se conocerá en el mundo por el nombre del pequeño pueblo al norte de Italia donde pasó su infancia: Caravaggio. De nada sirve, por cierto, que en nuestros días uno aprenda la existencia de otro pintor nacido en ese pueblo que se apropiaría antes del patronímico (Polidoro de Caravaggio, 1500-1543), porque es Michelangelo quien se ha llevado con él el símbolo y la leyenda.

¿Qué dirían de esta escena Ranuccio Tomassoni desde el infierno - adonde fue a parar luego de que fuera asesinado en una reyerta por el pintor de moda en Roma, que queriendo atravesar su sexo con la punta de la espada lo hiere mortalmente al abrir una vena vital de su muslo-, o su hermano desconsolado en una taberna romana, o el pintor y crítico de arte Giovani Baglionni, que detestara y envidiara hasta acusarlo ante los jueces y en su libro Le vite de'pittore, scultori et 
architetti al virulento Michelangelo, quien en un soneto se había burlado de él?

Quizás un paseante trasnochado o un pescador furtivo tropezó con el cuerpo lacerado por una espada ropera con guarnición en forma de lazo, con los ojos abiertos en dirección a Roma. Alguien tuvo que haber avisado al notario que escribiría después, apresurado, y con el año de diferencia del calendario de Siena: "A li 18 luglio 1609 nel ospitale du Santa Maria Ausiliatrice morse Michel angelo Merisi da Caravaggio, dipintore, per malattia".

Dos cartas de los archivos del Ducado de Urbino confirman que Caravaggio murió el 18 de julio de 1610 - casi dos años exactos después de ser proclamado en La Valeta Caballero de la Orden de Malta - cuando regresaba a su querida Roma desde Nápoles. El investigador Silvano Vincenti dio a conocer en 2010 que restos encontrados en una fosa común y que pertenecían al cementerio de San Sebastián correspondían a los del pintor. Vincenti afirmó entonces que una neurosífilis fue la causa de su muerte. Antes Vincenzo Pacelli, en su libro L'ultimo Caravaggio de 2002, especula sobre otra variante de la muerte del pintor: Caravaggio habría sido asesinado por la Orden de Malta con la aprobación del Vaticano.

Este final más caravagesco se avala por la más reciente conclusión de la ciencia. En septiembre de 2018 la revista Lancet Infectious publica el resultado de un grupo de científicos franceses que estudió la dentadura del pintor. Caravaggio no murió de sífilis ni de insolación, sino de una infección producida por la herida de una espada. Otra vez una espada. La espada que ocupa ya el centro de la luz en La vocación de San Mateo que él pintara en Roma entre 1599 y 1600.

Sobreviven de la vida de Caravaggio más incertidumbres que certezas. Por eso en el futuro, con toda seguridad, alguien propondrá otra vez nuevas versiones tanto sobre su vida como sobre su muerte. Como si su vida, al igual que los objetos y personajes de su pintura, estuviera velada, entreabierta, expuesta y escondida a la vez, entre luces y sombras de las que emergen como playas piel, rostro, mirada, siluetas iluminadas en la penumbra, y espadas, espadas que no pocas veces cercenan cabezas y siempre brillan, lustradas y viriles.

\section{Ante el espejo de Caravaggio y viceversa}

¿Qué nos hace volver a mirar un cuadro siglos después de haber sido concebido? Es la pregunta que me hago una vez más ante algunos de los 83 lienzos que se conservan de Caravaggio, los que he visto en Siracusa, en Madrid, en la Catedral de Toledo, en la National Gallery, en París (los tres del Louvre) y ahora en la exposición del museo Jacquemart André "Caravaggio à Roma", donde estoy contemplando Judit y Holofernes.

La cortesana Fillide Melandroni, en su papel de la viuda Judit, cercena el cuello del general Holofernes con una mano mientras con la otra hala el pelo del gimiente militar ante la mirada incitadora de una atroz y arrugada sirvienta. Atenta la sirvienta, con un costal entre las manos, lista para atrapar la cabeza arrancada. Los chorros de sangre escarlata que alternan con las cortinas plegadas del decorado saltan casi rectilíneos sobre la almohada a la que se agarra con sus manos de uñas sucias, o se tuerce cerrando el puño, el ebrio general asirio. 
Las formas de la tiniebla interrumpen el dibujo clásico de los cuerpos. El ambiente de contrastes de luces y sombras de la habitación y los objetos (la espada, otra vez la espada) concentra la atención sobre el acto fijado en el tiempo y la decapitación que será eterna llega desde una tumultuosa noche de trabajo romana hasta la pared iluminada de un museo del siglo XXI.

El espectáculo choca, aterra y atrae, hace cerrar los ojos o detiene la mirada al mismo tiempo. Se trata también de eso, de congelar el tiempo. Prima el espectáculo sobre la significación bíblica de la escena; cierta reticencia en el fruncimiento de cejas de Judit, los pezones que en una primera versión aparecían desnudos y ahora se yerguen puntiagudos bajo la blanca transparencia de una tela, las arrugas iluminadas de la criada y su ojo helado ante la excitación que le provoca la crueldad. Los personajes tienen una talla humana, pero están cortados para provocar, con la ausencia de paisaje y el encierro del espacio, una idea de profundidad que introduce al espectador en la escena.

Estamos de alguna manera ante Caravaggio. Y en esta contemplación del pintor entramos a la vez a su espacio privado y a su subjetividad. Formamos parte de una escena detenida en el momento más atroz de su ejecución. Estamos, hasta cierto punto, mirando al propio Caravaggio. El pintor y crítico Frank Stella llama "redondez" al espacio pictórico que crea Caravaggio.

La sensación de un espacio modelado que envuelve tanto la acción del cuadro como las posiciones del creador y del espectador, es efecto del poderoso realismo de Caravaggio. La sensación de estar ante una acción real y actual aumenta la sensación de espacio pictórico. Este es el primer milagro de Caravaggio, un milagro que con sorprendente economía de medios anticipa, y supera, los subsiguientes efectos ópticos del barroco.

Leon Battista Alberti, en su tratado De Pictura (1435), expone la tesis de que Narciso es el inventor de la pintura; "¿qué cosa es pintar sino besar la superficie de una fuente?", se pregunta. Édouard Pommier, en su libro Comment l'art devient l'art dans l'Italie de la Renaissance, afirma que Alberti inventa un mito fundador al identificar a Narciso transformado en flor en la pintura. "Pintar es reflejar", dice, "la pintura es una metamorfosis", y recuerda el adagio: "Ogni pittore dipinge se".

Giovanni Pietro Bellori (1613-1696), el primer biógrafo de Caravaggio - si no contamos al holandés Karel van Mander (1548-1606) - al criticarlo se apoya, entre otros juicios, en dos elementos que confirman el desdoblamiento del propio Caravaggio a través de sus lienzos. "Los cuadros de Caravaggio eran como él", dice Bellori con tono severo en su Vite de' pittori, scultori e architecti moderni (1672):

El estilo de Caravage estaba en concordancia con su fisonomía y su aspecto: tenía de piel sombría como sus ojos, las cejas y el pelo negros; y como tal aparece en su pintura. Su primera forma dulce y pura de utilizar el color fue la mejor, porque alcanzaba así un dominio supremo y se muestra, ante los elogios de todos, como un excelente colorista lombardo. Pero pasa enseguida a un estilo oscuro llevado por su temperamento tormentoso y pendenciero, por lo que tuvo que dejar primero Milán, su patria, y debió huir de Roma y de Malta hasta esconderse en Sicilia y afrontar una existencia peligrosa en Nápoles hasta encontrar en una playa una muerte miserable. 
El segundo elemento completa una desconcertante paradoja: Bellori le critica a Caravaggio que utilizara espejos para pintar directamente sin dibujos previos: "Hizo algunos cuadritos de sí mismo retratado en el espejo. Y el primero fue un Baco con algunos racimos de uvas diversas". ¿Si la obra es copia del artista y de su atormentada existencia, por qué criticar que se valga de un espejo?, me pregunto.

Roberto Longhi en su clásico libro Caravaggio de 1952 argumenta que en realidad utilizando un espejo Caravaggio respeta a su manera la antigua exigencia según la cual la pintura debe ser un reflejo de la realidad, pero limitándose a la imagen que él veía en el espejo, la cual era su realidad objetiva. "No se trata ni siquiera de un trompe-l'oeil", dice Longhi, "porque la imagen que propone Caravaggio es natural y opuesta a un retrato".

Los estudiosos del lombardo han ido detectando varios autorretratos del pintor. El Baco enfermo (1593) ya citado, el hombre del fondo que mira al espectador en El martirio de San Mateo (1600), un espectador que mira hacia la luz en El entierro de Santa Lucía (1608), y la autorrepresentación de Caravaggio: David con la cabeza de Goliat de 1610. La cabeza de Goliat, con la marca de la pedrada de David en la frente, es en realidad un retrato de la cabeza de Caravaggio. En la espada de David aparece una frase de San Agustín como epitafio: "Humilitas occidit superbiam" ("La humildad mata al orgullo"). Aquí Caravaggio se representa como el mal y la cicatriz en la frente es también la herida que sufriera en Nápoles poco antes de morir: la herida de una espada. David aparece remedando a Temis, la Justicia, solo que en lugar de la balanza sostiene en una mano la cabeza del pintor; no obstante, en la otra lleva la espada de rigor.

Pero sin dudas la más sorprendente autorrepresentación del pintor es la descubierta en 2014, en el Baco de 1595. Más que la figura andrógina del dios, Ilaman la atención los objetos. Tras la transparencia del vino de la jarra que aparece a la izquierda del espectador, es decir, a la derecha de Baco, se pudo detectar un autorretrato del pintor después de utilizar una técnica nombrada reflectografía multiespectral.

Caravaggio aparece ahí, dentro del vino, pintando con un pincel en la mano y "grandes cuencas oculares, una nariz ancha y ligeramente tersa y labios carnosos y ligeramente separados". El espejo es el vino, o viceversa.

\section{Espadas y sotanas}

La leyenda de sus escándalos persigue a Michelangelo. Tanto lo persigue que se habla más de su vida que de sus cuadros. Pero nada es falso: cuentan que después de pintar todo el día vagaba con su espada por los antros de la noche romana. Uno lo imagina volviendo a su taller después de las juergas con una convencida amiga cortesana que aceptara posar: sus cuadros parecen salir de la noche empujados por una insólita luz que atraviesa desde un ángulo inesperado a los cuerpos y las uvas, los rostros, las miradas y la tela de las ropas de sus personajes. Frank Stella escribe sobre esta atmósfera imaginaria:

Y tras tocar los objetos, nos fijamos en los personajes, en las figuras. ¿lban los modelos de un lado para otro de la escenificación del martirio, hasta que Caravaggio daba la señal 
y se quedaban congelados? ¿Corrigió cada pose teniendo en mente a Rafael, Tiziano o Tintoretto? ¿Cuánto tiempo quedaba congelada la pose? ¿Cómo dirigía todo eso? ¿Lo haría como Mel Brooks o como Ingmar Bergman, como Fassbinder o como Fellini? Poco en claro podemos sacar de las elucubraciones literarias que pueden hacerse sobre el taller de Carvaggio; sólo podemos remitirnos a los cuadros.

¿Qué, además de su talento, puede explicar su genio? ¿Qué lleva al célebre crítico inglés Roger Fry - a quien Virginia Wolff le dedicara una monografía - a afirmar que "él fue el primer artista moderno, el primero en proceder por una revolución y no por evolución"?

Su lista de mecenas es extensa, pero se cita a un solo preceptor. Comienza de aprendiz en Milán, en el atelier de Simone Peterzano (1540-1596), antiguo discípulo de Tiziano. "S. Petrazanus Titiani Alumnus", le gustaba firmar a su maestro. Existen pruebas de que su madre - que moriría poco después dejándole una pequeña herencia con la cual puede costearse el viaje a Roma en 1592paga la formación a Peterzano. Nada queda de sus dibujos (él que casi no dibujaba sobre la tela antes de las pinceladas) de estos cuatro años. Uno de sus primeros críticos, Karel van Mander (1548-1606), en su Het Leven der Moderne ofd dees-Tijtsce doorluchtighe Italiaenische Schilders, Alkmaer, de1603, denigra a Caravaggio por pintar desde la naturaleza misma que ve y sin dibujo.

La influencia de la escuela veneciana y la lombarda se citan cada vez que se pretende especular sobre ese claroscuro que terminaría adoptando el nombre de caravagismo. De él vienen (muchos lo olvidan) Zurbarán (el Caravaggio español), José de Ribera, Rembrandt, Rubens, Georges De La Tour, y muchos otros.

Pero Caravaggio, a ratos también poeta y músico, no es solo el bohemio virulento admirado y vilipendiado en toda Roma, sino también el alumno discreto que hace suyas las ideas predominantes en los círculos culturales de moda, todos, cercanos a las autoridades eclesiásticas. Mucho se ha insistido en los preceptos ideológicos y estéticos de la Contrarreforma y del Concilio de Trento para fundamentar la originalidad simple y real de sus personajes religiosos y paganos.

En Milán pasa su juventud en una familia que lo tiene al tanto de las ideas del obispo Charles Borromée, discípulo de Ignacio de Loyola y Teresa de Ávila. Por su parte, el repaso de la extensa lista de mecenas y coleccionistas que le encargan obras, no tarda en convencernos de que, como la paradoja que fue su vida, el pintor de Joven tocando el laúd (su obra preferida), muerto anónimamente en una playa casi desconocida, llevó siempre consigo en sus errancias, junto a sus pinceles y su espada, la protección y los consejos de las sotanas.

\section{Judith en un desván de Toulouse}

En una casona del siglo XVII en las afueras de Toulouse, a principios de 2014, los dueños (una vieja familia de esta ciudad francesa) le ruegan a un plomero portugués encontrar las causas de un persistente salidero. El goteo viene del techo debajo del cual hay un desván. El plomero pide permiso para derribar una pared de madera, porque todo parece indicar que el origen del salidero está detrás de ella. 
Imaginemos la sorpresa, la curiosidad y el asombro al tener ante sí una versión desconocida de Judit y Holofernes, el célebre cuadro de Caravaggio. La escena es similar con algunas diferencias. Solo aparecen de nuevo los tres personajes: Judit, Holofernes y la criada que espera, impaciente, por la cabeza cercenada con una especie de saco entre sus manos. Lástima, dirá uno de los detractores en medio de la polémica que comenzará días después, que las arrugas de esta vieja diabólica no estén ensombrecidas a la manera en que lo hacía el genio lombardo. Detalle este que contradice el americano Benjamin Sutton, quien compara a la vieja del cuadro con la de La crucifixión de San Andrés del Museo de Cleveland: son idénticas.

Es un hecho (comprueban los genealogistas) que la familia de Toulouse desciende de un antiguo general de Napoleón, y hace un tiempo ya han subastado el cuadro de un pintor español adquirido en sus contiendas por este ilustre antepasado.

Todo se precipita. Se trata nada más y nada menos que de un cuadro cuyo valor, corriendo, se estima en unos 120 millones de euros. El 14 de abril de 2014, el experto y subastador parisino Éric Turquin recibe en su gabinete el cuadro y piensa enseguida que se trata de un Caravaggio perdido. Su entusiasmo no tiene límites ("para alguien al borde de la jubilación como yo este cuadro es un regalo sagrado", afirma) y descubre dos o tres detalles que pretenden demostrar la autenticidad del lienzo: las uñas sucias de Holofernes, trazo de identidad del pintor que quiere insistir en este detalle de la mundanidad. Y un dedo de la mano varias veces pintado. "Un copista" (este es el argumento principal de los negadores: el cuadro es una copia, probablemente del pintor Louis Finson, quien ya había reproducido el cuadro, como lo muestra el conservado en el banco Intesa Sanpaolo de Nápoles), dice, "no se detiene a corregir detalles".

Después de varios análisis, el 25 de marzo de 2016, el Ministerio de Cultura de Francia exige que el cuadro no salga del territorio francés por considerarlo patrimonio cultural.

Entre el 7 de noviembre de 2016 y el 6 de febrero de 2017, los expertos se reúnen y confrontan el cuadro de Toulouse en la pinacoteca de Brera, en Milán, con Los discípulos de Emaús (del cual existe otra versión que yo he visto en la National Gallery de Londres), sin que se llegue a acuerdo alguno. Los negadores terminan por ganar. El estado francés declara el 11 de enero de 2019 que no retendrá la venta pública de este cuadro, por haber dudas sobre su autenticidad. Una subasta se organizará por los tres herederos del general de Napoleón en Toulouse, siguiendo al parecer una moda reciente: la de rematar las obras en lugares descentrados de las capitales del arte para eludir les enormes comisiones cobradas por las casas de venta tradicionales como Christie's y Sotheby's.

$Y$, sin embargo, todos los análisis de laboratorio coinciden en la autenticidad de los pigmentos y el lienzo. Los rayos $\mathrm{X}$, incluso, ven similares retoques en la tela de Toulouse y la de Milán, sobre todo en las miradas de la criada y de Judit.

Eso sí, en ambos cuadros Judit empuña la espada en el momento de la decapitación; pero en la versión de Toulouse la espada se yergue al centro sostenida por la luz lateral que penetra en la escena congelada. Pocos parecen saber que la modelo es la misma Fillide Melandroni que fuera amante de aquel Ranuccio Tomassoni a quien Caravaggio ultimara en Roma de una estocada equivocada, provocando con esa tragedia el comienzo de su propio fin en una playa desierta. 
